\title{
Belphégor
}

\section{De la couverture illustrée du roman populaire}

\section{Charles Grivel}

\section{(2) OpenEdition}

\section{Journals}

Electronic version

URL: https://journals.openedition.org/belphegor/1270

DOI: 10.4000/belphegor.1270

ISSN: 1499-7185

\section{Publisher}

LPCM

\section{Electronic reference}

Charles Grivel, "De la couverture illustrée du roman populaire", Belphégor [Online], 16-1 | 2018, Online since 16 July 2018, connection on 19 October 2021. URL: http://journals.openedition.org/belphegor/ 1270 ; DOI: https://doi.org/10.4000/belphegor. 1270

This text was automatically generated on 19 October 2021.

\section{(c) $($ ) $(9)$}

Belphégor est mis à disposition selon les termes de la Licence Creative Commons Attribution - Pas d'Utilisation Commerciale - Pas de Modification 4.0 International. 


\title{
De la couverture illustrée du roman populaire
}

\author{
Charles Grivel
}

\section{REFERENCES}

Parution originale dans Jacques Migozzi et Philippe Le Guern (dir.), Production(s) $d u$ populaire, Limoges, Presses de l'Université de Limoges, « Médiatextes », 2005.

1 1. La couverture d'un livre est cet objet rapporté, adjacent, accessoire, artificiel, apparemment futile, en tout cas secondaire, auquel nous avons été pourtant quelquesuns - Gérard Genette, Anne-Marie Thiesse, Daniel Couégnas, Philippe Hamon, Marc Lits, moi-même - à attacher de l'importance : un livre est " couvert », ce qui veut dire que sa matière se dérobe et aussi se camoufle, quoiqu'elle se manifeste. Tout le paradoxe est là. Ce que nous recevons à lire possède cette particularité de devoir être assimilé avant que d'être saisi, sa reconnaissance se doit d'être, forcément, préliminaire, puisqu'il en va de l'acte d'achat. Il faut avoir lu avant que de lire. Il convient d'être saisi par l'objet de sa lecture, alors même que celle-ci n'a pas pu débuter. La «couverture »- qui possède, certes, plusieurs faces, un «dos», une " quatrième », une «bande », une « jaquette » - constitue donc le passage obligé grâce auquel un lecteur se représente le livre, imagine être informé sur son contenu, adapte son attente, évalue les chances de satisfaction, décide de passer à l'acte. À moins, bien sûr, que l'incitation ne vienne d'ailleurs, d'une obligation scolaire, d'une autorité parentale, des circonstances. Or, sur le plan qui nous concerne - la fiction littéraire et populaire - , la couverture joue pleinement son rôle d'aimant: nous y regardons comme en un miroir ou en un gouffre l'image du désir que le livre propose.

2 Une image "couvre", ainsi, le livre. On conserva, certes, vierge, dans les premiers temps, la page d'ouverture, on fit aussi l'économie du feuillet réservé au titre, le début $\mathrm{du}$ livre fut "aveugle», comme on dit, et le lecteur se trouvait confronté, sans intermédiaire, à la matière même de l'ouvrage : dans un monde où le livre était rare, 
cela n'offrait pas d'inconvénient. Mais le développement de la librairie, dans les temps modernes, imposa de nouvelles habitudes: il fallut signaler au chaland - au bibliothécaire - au collectionneur - de quoi l'ouvrage était fait, sur le dos et sur l'àplat. La "couverture» devint alors un support à part entière d'informations nécessaires à son appréhension, elle comporta bientôt de quoi en manifester l'excellence, l'intérêt ou la valeur. Cette icône en lettres majuscules, sur papier coloré, comportant souvent ou un dessin ou un insigne, à la fois insignifiante et captivante, illustration pourtant non illustrative, chargée de donner au livre, au récit qu'il contient, aux personnages qui s'y déplacent, une apparence conjecturale efficace, se posa comme l'accompagnatrice obligée du livre. Elle le parla. Elle le représenta. Nous, lecteurs, allons nous approprier celui-ci en même temps que celle-là. Nous serons riches de deux, mais divisés, déjà, dès le premier moment, dans nos attentes.

Dehors manifeste et couvercle mis à une chose sans nom, invisible encore, présentement renfermée dans une sorte de boîte oblongue - un "volume » - une bière - une histoire -, inconnue pour l'instant, impénétrable pour un temps, rendue, néanmoins, grâce à la face colorée qu'elle montre aimablement, comme un fard, infiniment désirable. Regarder par l'ouverture du livre est devenu nécessaire, que disje?, nourrit, désormais, une satisfaction supplémentaire au livre, comme une prime. Je vois ce qui se passe au fond du corridor des lettres. Descendu là par une bouche propice, je me déplace, tel Marius dans les égouts de Paris, je me désole comme le pantin devant sa grille à considérer là hors de portée les Paquita de tous les romans. J'aime ce que je ne reçois pas, pourvu qu'on me le promette.

4 2. Mon titre est approximatif. Il faudrait mieux dire "Couverture-ouverture et image de garde » - pour marquer l'équivocité de la fonction -. « Les couvertures illustrées de la littérature policière des années cinquante » - l'infinité du corpus ayant de quoi effrayer, le cas choisi aura valeur de paradigme.

5 Je m'occupe donc d'un phénomène, si l'on veut, microscopique : la présence d'une page spéciale, inclue au volume, marquant celui-ci à une place privilégiée et lui assignant un certain nombre de caractéristiques propres à en faire connaître la nature ou le sens au lecteur. Propres aussi à en accroître la désidérabilité. Du moins, si l'on tient compte de l'évolution des fonctions que connaît la couverture au cours de son histoire, car cette inscription du caractère désirable du livre à son entrée est tardive. J'inscris, en effet, mon étude, très précisément, dans le cours récent de cette histoire et m'attache à cerner la forme et la valeur qu'elle prend dès lors que le livre de fiction - «littéraire » ou " populaire " - devient, dans le courant du dix-neuvième siècle, "à la devanture ", "sur le tourniquet du kiosque", «à l'étalage du libraire», un objet offert à la consommation, voire à la concupiscence du client. Cette banale exposition du livre par son emballage, imposée par la société de marché change tout - l'achat, l'attente, l'affect. Désormais, nous lirons aiguillonnés par un désir qu'un élément rapporté, iconique souvent, définit : ce n'est pas le livre qui prend la parole à la couverture, un bâillon typographique illustré, dirons-nous plutôt, lui est infligé.

6 Un livre possède, en principe, une " première page ", une page « hors jeu » « de garde ", une " couverture ", doublée ordinairement de celle qu'on appelle la " page de titre » quand elle ne l'est pas encore d'un «frontispice » qui propose de manière plus sobre, mais plus précise, toutes les informations souhaitables concernant l'auteur, l'éditeur, la collection, la date de parution, etc., ainsi que l'intitulé complet de la publication. Cette page d'accès, par où le lecteur est introduit dans le monde du livre, devient l'objet d'un 
traitement privilégié de la part de l'éditeur, à partir du moment où le volume doit être exhibé pour être acquis et donc montré à un public qui n'en a pas, par avance, la notion suffisante. Ce phénomène dérive de ce que j'appelle la loi de la devanture.

7 Une précision, pour commencer : parmi toutes les fonctions que peut remplir, depuis les temps lointains qui virent la naissance du livre tel que nous le connaissons, la «première page » du volume - devenue «couverture » - je n'en examinerai, avec quelque soin, qu'une: la fonction d'ouverture. Par l'un de ses principaux aspects, en effet, la couverture s'articule au contenu propre de l'ouvrage - ici, de fiction - et en scelle, d'une certaine façon, le sens et l'usage. Je ne m'interroge pas, ici, sur les valeurs attachées au " brochage » ou à la « reliure » - d'art ou bibliophilique -, sur la nécessité de "protéger " l'ensemble du volume par un emballage approprié, sur l'obligation d'informer le public sur l'identité de l'auteur, la nature de l'ouvrage, le genre duquel il relève, l'éditeur qui le publie sous sa responsabilité, etc. Je ne m'interroge pas sur cette instrumentalisation-là de la couverture.

8 Je pointe, par contre, la dimension à la fois projective (elle "introduit ») et rétensive (elle "oriente ») de la couverture - sa fonction d' "ouverture». Cette fonction, pour l'essentiel et pour l'époque qui nous intéresse, est prise en charge par l'illustration. C'est donc de couverture illustrée dont je vais vous entretenir. C'est-à-dire, de cette page inaugurale, d'abord et en principe « hors le livre », qui petit à petit va s'y intégrer et s'y intégrer pleinement, dès lors qu'elle devient une image, et parle, de ce seuil, en réserve de ce qui va être dit, prédictivement. J'examine un emploi, relativement récent et relativement particulier, de ce traitement. Je sais que l'insertion de la page aveugle (ou semi-aveugle) du titre (ou de l'emballage) démarre, fort tôt, dans l'histoire de l'imprimé avec la pratique du «frontispice », puis de l'illustration « hors-texte » intercalée entre la couverture proprement dite et la page de titre (intérieure) du livre, puis avec celle de son implant page une de la livraison illustrée, au milieu du dix-neuvième siècle. Je sais qu'il a fallu du temps pour que l'image vienne occuper sans complexe - sa réputation ayant toujours été douteuse - la face d'entrée du volume. Mais je sais aussi que l'image a toujours été déjà là, ne demandant qu'à paraître, sous la lettre, miniaturisée, dans les marges, comme décor, entre les lignes, de couleur en couleur, puis comme illustration, potentielle et désirée. Elle semble avoir émergé d'un texte conçu dans un lointain imaginaire qu'elle aurait pour mission de reprocher.

9 En tout cas, c'est mon observation de base, le récit tend à remonter à la page 1 - à la page de couverture - inauguratrice du volume, voire à l'envahir dès qu'il se range à la logique industrielle; ce récit s'en sert alors comme d'un tremplin, comme d'un promontoire donnant sur ce qui fait sa matière, permettant d'accéder à la lecture proprement dite. Je ne retrace pas les étapes qui ont mené, jusqu'aux années cinquante, à la forme prise par le phénomène : les ingrédients, les caractères, les styles mis en œuvre par la couverture sont multiples et à peu près tout reste à faire sur ce terrain - je ne fais que débroussailler. Je constate seulement que la massification de la culture a trouvé un second souffle avec l'introduction sur le marché du livre de poche : le "livre de poche " à la française, dans le cadre duquel mes observations s'inscrivent ici, reprend les recettes qui ont fait leurs preuves, avant guerre et précédemment aussi, sur le circuit des petits romans: de la couleur (mais maintenant sur papier glacé), de la naïveté voulue ou une certaine approximation attractive dans le trait, de l'expressivité (que le dessin souligne). Un certain jeu sur la perspective, sur la "prise de vue», héritée du cinéma se fait jour, certes, mais, pour l'essentiel, la règle reste inchangée : 
faire entrer, de plein-pied, le lecteur dans le drame, indépendamment de l'ignorance où il est encore de sa nature réelle. Qu'elle obéisse ou non à une stratégie commerciale déclarée, la couverture a quelque chose de la fausse étiquette.

Une remarque encore, à laquelle il conviendrait de donner plus d'ampleur : le «style » de la couverture fixe l'attention sur une collection et fidélise le public. Plus on s'élève dans l'échelle des valeurs littéraires, plus la réputation et la signature du dessinateur devraient être, à cet égard, porteuses. Pourtant, on observe que la littérature haut de gamme boude la couverture tapageuse et il n'est pas certain que les noms des prestigieux $^{1}$ dessinateurs des séries populaires (Starace pour les romans d'aventures de Fayard, Gourdon pour les séries populaires de Fleuve noir, Nicollet pour la collection "Néo » de chez Oswald) soient réellement identifiés par la masse des lecteurs. Peu importe, cependant : même anonymes et sans valeur artistique, le style et le genre du dessin de couverture, sa couleur - aussi laide soit-elle -, son « logo» - aussi puéril qu'on voudra -, ses tics et ses stéréotypes sont susceptibles de remplir leur office, des années durant : unifier dans la variété, composer avec un matériau restreint un signal unique pour une entrée multiple. Faire signe est ce qui doit suffire.

11 3. Cet objet « futile », on le voit déjà, n'est pas un objet simple. La couverture, ensemble composite, bien qu'elle fasse usage du titre de l'ouvrage et aussi du nom de l'auteur, présente ceux-ci sous un éclairage soigneusement calculé : le premier est souvent simplifié et la typographie choisie - les majuscules, les lettres de fantaisie, sert plutôt à susciter des virtualités narratives qu'à simplement « informer » qui de droit; quant au second, il est tenu dans une relative pénombre et la modestie des caractères qui sont les siens en dit long sur l'importance que joue, dans ce circuit, sa réputation ${ }^{2}$. De la même manière, lorsqu'elle requiert une illustration, l'auteur n'en est que, dans quelques cas, précisé : la couverture est cet espace anonymisé, simplifié, schématisé, rendu propre à engager une lecture que la complication (des faits, des sens) ne manquerait pas de rebuter. La lecture s'engage sur un terrain préalablement nettoyé, propre à faire émerger quelques icônes, quelques signaux, chargés de déclencher l'affect, et à leur donner tout le relief nécessaire.

Il vaut la peine de procéder ici à un petit bilan de la recherche : on mesurera mieux, ainsi, la nature nécessairement plurielle, hybride et fugitive, de cette affiche du livre et aussi quelles sont les difficultés qui surgissent, dès qu'il s'agit d'en faire l'analyse. AnneMarie Thiesse insiste bien sur le caractère "attractif » de la couverture du roman populaire, avant et après la Première Guerre mondiale, puisque tel est son objet et note le passage du monochrome au polychrome : une " séductivité " accrue, non seulement de l'image, mais du fond sur lequel elle se détache, est à ce prix ${ }^{3}$. Gérard Genette, pour sa part, excelle à dresser l'inventaire de tout ce qui peut se marquer à la couverture, comme "péritexte éditorial ${ }^{4}$. Il néglige largement, pourtant, sa matérialité et son caractère illustratif - il note simplement que l'illustration de la couverture est "spécifique ", ce qui est vrai - et se borne, on le comprend - à relever son caractère "voyant» et «citatif» (dans le cas des adaptations qu'il évoque) ou alors « individualisateur» (dans le cadre nécessairement uniforme de la collection). Un " texte sans paratexte ", note-t-il non sans humour, est "parfois comme un éléphant sans cornac »; inversement, le "paratexte sans texte " évoque l'idée absurde d'un « cornac sans éléphant » - « parade inepte », s'il en est, dit-il ${ }^{5}$, imaginaire creux dont la profondeur reste insondable. 
Philippe Hamon, quant à lui, voue son attention, au chapitre VII d'Imageries ${ }^{6}$, au "frontispice ", auquel il reconnaît, à juste titre, des vertus cardinales qui sont aussi, souvent, celles de la couverture, dont il ne parle pas en propre. Le frontispice « expose » le livre, il revient à cette « image-seuil » de résumer l'ouvrage, de lui servir d'enseigne ou de quasi "générique »; elle ouvre, pour le lecteur, «l'horizon d'attente imaginaire du texte qui va suivre » : « l'acte de lecture s'inaugure par un acte de regard qui construit, via le frontispice, une image virtuelle et prospective du texte à découvrir». Or, cette vertu "allégorique», cette fonction de «matrice», cette "prégnance ", cette sorte de mise en scène au début du livre, tout au cours du dixneuvième siècle, $d^{\prime}$ ' un art poétique déguisé ", plus ou moins perceptible ou concluant, ne remplit pas à lui seul, bien sûr, tout ce programme - il n'en est, à vrai dire, que l'indice : c'est par la couverture que s'inaugure, à mon sens, réellement, la lecture et c'est grâce à celle-ci, d'abord, que le lecteur accède au "montrer-dire» du livre. Au centre d'un péri-texte et d'un para-texte complexes et multiples comprenant, outre le frontispice - rare, dans les productions ordinaires, au demeurant - , le portrait de l'auteur, la préface et la dédicace, l'exergue et la page de titre, la liste des ouvrages du même auteur et des feuillets de réclame - et bien d'autres choses encore -, la couverture concentre en elle-même et sur elle-même, immanquablement, de quoi lire. Elle n'est pas, comme le frontispice, un message supplémentaire au livre et n'en ordonne pas la matière, mais elle fait entrer en combustion sa substance et "chauffe » ses motifs, manifestes ou secrets ; l'image qu'elle organise ouvre la porte à l'imaginaire, nous voici, déjà, de I 'autre côté du miroir.

Marc Lits, pour sa part, dans un travail plutôt pragmatique déjà ancien, mais publié récemment ${ }^{7}$, propose un petit tour d'horizon de la question et appelle de ses vœux une étude systématique, nécessairement coûteuse, remarque-t-il, et il n'a pas tort, de la dimension "herméneutique» de la couverture illustrée ${ }^{8}$. Il relève la "potentialité narrative » de l'image de garde et note que son rapport d'« indicialité » au texte qu'elle couvre pose problème. Il pointe, enfin, la fonction réceptive qu'elle accomplit dans la fidélisation d'un public dont il faut retenir l'attention. Ce sont là des dimensions à explorer. En ce qui concerne la nature de la couverture en tant que telle, Marc Lits remarque, lui aussi, son caractère « criard » et « stéréotypé ».

Enfin, Daniel Couégnas s'est intéressé, voici plus d'une dizaine d'années', aux couvertures de la série Fantômas illustrée par Gino Starace pour la collection « Le Livre populaire » de l'éditeur Fayard. Son étude, assez fouillée, pointe, le contraire eût été étonnant, la fascination exercée - dans ce cas seulement ? - par le travail du génial illustrateur ; il voit en la couverture une « affiche publicitaire » en réduction du livre, il en note certains éléments, relève la composition "cinématographique » de l'illustration - dans ce cas ? à cet endroit seulement ? - du dessin de couverture et insiste sur la nature du poncif qu'elle constitue. Voilà, dit-il, c'est une remarque dont je me souviendrai, un bon « judas » par où viser la future matière du livre : le délai de la vision - et l'impunité du voyeur - est, en effet, la clef de son fonctionnement.

Cependant, pour intéressantes que soient ces études, il me semble que nous sommes encore assez loin du compte: la couverture illustrée comme mécanisme visuel d'entraînement à lire reste énigmatique et même si ma contribution d'aujourd'hui demeure, elle aussi, fragmentaire, c'est cependant ce mécanisme global de visionnement que je voudrais contribuer à éclairer ${ }^{10}$. 
17 4. Je reprends. En effet, on ne peut se borner à définir matériellement un livre comme un certain nombre de feuillets imprimés simplement cousus ensemble pour convenir à la commodité du lecteur. Un livre est encore le simulacre de l'objet livre, c'est-à-dire une entité qui représente ce pour quoi elle se donne en sus de son contenu effectif. Il consiste en ses pages imprimées signifiées à la page de garde. Il ne peut éviter de s'ériger comme livre, afin d'être consommé. Il doit être rendu visible. Il doit être cru, demandé. Il doit se désirer. Plaire, autant que possible. Or, le livre est cet objet particulier, qui, dès lors qu'il entre dans le circuit de la consommation, cherche à être demandé. Pourtant, la connaissance n'en est possible qu'après coup, il faut se l'être approprié pour en mesurer vraiment la nature et la valeur. Il fallait donc trouver le moyen de signifier a priori son contenu, de profiler hors cadre son propos, d'indiquer la donne avant que d'entamer la partie. La couverture a donc à voir avec la désidérabilité du livre, étant donné que la saisie de l'objet - du récit, du sens - ne peut s'en faire qu'après coup : comme un vêtement, il ne peut convenir qu'après essayage. Cependant, un livre, lui, ne s' « essaie " pas. Mettre une couverture à un ouvrage, c'est réussir cette (banale) gageure de rendre plaisant ce dont l'idée est présente, mais ce dont la vérification actuellement ne peut que manquer. La première page, convenablement préparée, suppose le livre ; elle représente le désir qu'il est censé alimenter ; elle pousse à sa croyance ; elle conduit à sa nécessité.

La couverture simule, par conséquent, d'une façon ou d'une autre, le contenu du livre et projette celui-ci «à plat», matériellement, signalétiquement, sur le plan-cadre géométrique du volume. Cette feinte référentielle est fondatrice et fait de la couverture illustrée un index avant que d'être une image, un «dessin », la représentation de quoi que ce soit qu'on voudra. "C'est une loi irréversible que tout livre, écrit Claude Maggiori, s'il veut dorénavant se positionner sur le marché, doit être porteur d'une certaine image de marque. C'est-à-dire qu'en allant l'acheter, le consommateur doit non seulement déjà avoir un aperçu de ce qu'il contient, mais également pouvoir imaginer l'allure que ce livre lui donnera en société ou les réactions qu'il suscitera auprès de ses proches $»^{11}$. Cette dernière réflexion, moins insignifiante qu'il ne paraît, ne doit pas étonner: un ouvrage fait signe sur celui qui en est le possesseur, il l'accrédite, il lui confère, petite ou grande, une certaine aura, ou, du moins, une prééminence, une priorité, un privilège, la valeur qui s'attache à l'antériorité. Et c'est ici encore que la couverture illustrée - avec ou sans image, vierge de fantaisie ou colorée - a son rôle à jouer : elle parle, non seulement en direction de l'ouvrage, mais aussi en direction de celui qui vient de l'acquérir. Elle l'honore ou pas. C'est un petit drapeau qu'il promène avec soi, qui signifie son parti, sa culture, de quelle génération ou idéologie il relève, etc. La couverture a beau être «fausse » par rapport au livre, elle réfère pourtant - à la masse inerte, encore, du texte, à la qualité du lecteur, elle le flatte en même temps qu'elle le séduit. Elle manifeste une question qu'en somme elle formule à elle seule (que peut vouloir dire la chose à lire ?), mais elle claironne aussi la nature de celui auquel elle convient. En d'autres termes, un principe classificateur agit en elle.

19 Ainsi, la couverture pose-t-elle le livre, à la fois comme une essence dont elle serait la manifestation et comme un contenu naturellement problématique, dont le sens se dérobe, tandis qu'en fait, miroir aux alouettes, elle figure son lecteur, ses attentes et ses désirs, et les imprime, sous ses yeux médusés, sur l'innocent volume. 
20 Cette image à la couverture - cette couverture comme image - imagée ou non typographiée, décorée et colorée, avec tous les inserts informatifs qu'on voudra, nécessairement, ment : sur ce dont elle parle (le texte), sur son appropriateur, sur ellemême; ce qu'elle indique n'est rien de ce qu'elle indique; sa puissance réflexive est majeure et cependant naïve : plus elle " parle ", plus on ne la saisit pas. La couverture « comme image » est donc un faux, elle ne répond pas réellement à son contenu putatif. L'image qu'elle offre - dans son libellé, ses bandeaux, ses logos, ses dessins, car ils sont indissolublement liés - se lit moins comme telle dans le pur aspect qu'elle offre, que dans le signe qu'à eux tous ils forment: peut-être ne voyons-nous rien de la belle apparence platinée et glacée du papier, sinon qu'elle nous méduse et nous entraîne dans le flot de son Rhin imaginaire - nous ses pêcheurs. Ce qui est dit ici ne l'est pas, en vérité : comment déchiffrer un signe donnant sur une particularité inconnue de celui qui en détient pourtant la clef ? C'est le moins qu'on puisse dire : la couverture se nourrit des contradictions qui l'inspirent et nous en voyons, en somme, à la considérer, toujours un peu trop et toujours pas assez. Le rapport est celui d'une robe à un corps pour l'amant : c'est dans l'intervalle ménagé là entre ce qui apparaît et ce qui n'apparaît pas - pour le voyeur - qu'il faut aller chercher son charme. Retenue, d'un côté, affiche, de l'autre, rien, peut-être, et, cependant, tout : à quoi obtempérer sinon?

21 Je réfléchis à la nature du dispositif. La couverture est illustrée. Mais l'image qu'elle offre - qui ne contient, d'ailleurs, pas nécessairement ce qu'on entend généralement par image - un dessin, une forme, quelque chose de dramaturgique -, est une image impossible : son objet n'a pas d'équivalent dans le texte, ce que vous voyez manifesté ne possède pas d'existence " hors là » - c'est un objet absent, à quoi, pourtant, beaucoup, sinon tout, dans l'histoire, maintenant désirée, conduit.

22 A la limite, la couverture de la sorte que je dis se suffit à elle-même, comme un étendard. tandis que le livre, s'il est finalement ouvert, se présente plutôt comme son appendice ou son spectre: la couverture est (contient ou retient en elle-même) le principal motif de la lecture, son "accroche", le livre se range sous la perception rétrospective évanescente qu'elle en permet ; il lui apporte substance et matière, bruits et mystères, fait vibrer des appréhensions qu'elle a provoquées ; confirmation jamais démentie, dénégation jamais consentie, il s'exprime, par échos, dans le discours, approximatif, qu'elle lui impose de tenir.

23 Je constate que, dans notre système médiologique consumériste moderne, tout ce qui est offert à la libido doit être " couvert », protégé, emballé, plastifié, marqué, désigné, nommé, protégé - et illustré - pour être compris, ou plutôt assimilé, et que toute expression, pour séduire, doit être doublée d'une autre ou accompagnée d'un fairevaloir qui en magnifie a priori les performances. Un pot de confiture nécessite une étiquette, un médium sollicite un autre médium afin de pouvoir faire entendre le langage qu'il cherche à tenir. La couverture est ainsi un dispositif pervers : elle instruit de ce dont la connaissance manque, elle manifeste ce sur quoi le regard achoppe, elle alimente la compulsion, plus ou moins fébrile, du livre par le lecteur, dirige son œil sur le point qu'il cherche, mais qu'il ne rejoindra pas.

Béance, exténuation de la demande, à plat des intervalles, creux déployé sous les pas du lecteur aventureux : j'interroge le sans-fond d'un mécanisme alternatif à deux coups, le premier ouvre une attente, le second met un terme, ou s'efforce de mettre un terme à sa nécessité. Je crois, certes, que le récit ne contient pas réellement de quoi répondre à la demande formulée à la couverture, mais je crois aussi qu'il n'est pas question qu'il se 
soustraie à l'obligation qu'elle lui fait. Il est, ce récit, ce roman, ce texte, comme la face éclairée du message, tandis que nous en considérons, d'entrée de jeu, sur cette image que rien, présentement, n'assure, la face obscure. Cette incommodité n'en est pas vraiment une : comme dit Gérard Macé - avec Victor Hugo « la mémoire aime chasser dans le noir $»^{12}$.

La couverture illustrée, battement d'incertitude imposé dès l'abord à la matière écrite, constitue ainsi le livre en un mécanisme pervers. C'est mon interprétation. On dira que c'est employer là un bien gros mot. Mais qu'on y réfléchisse : ce qui est montré, proféré, en un clin d'œil, à la couverture, par une image, comme cette barrière de chair blanche qu'une jarretière opportunément découverte découpe au bon moment sous cela qu'on ne verra pas, est tenu sous voile par la suite - car un récit (puisque c'est dans cette sphère où nous nous situons) ne montre pas, ne parvient pas au but de révélation auquel il tend - la structure de l'histoire policière l'indique parfaitement, mais aussi le feuilleton, le roman à suivre, les séries romanesques, la pulsion d'un récit à la Dumas, chapitre après chapitre, ou encore les annuités ponctuelles, forme sous laquelle se présentent les publications zoliennes. Dans la mesure où la vision est promise, dès l'ouverture, par un dessin qui l'atteste, le corps du texte ne peut plus que se montrer déceptif. Nous finissons par savoir qui a tué, mais nous ne verrons pas, et à jamais, la scène capitale à laquelle, par calcul, la couverture nous promettait d'assister.

«Couvert» de la sorte, un livre est devenu un objet paradoxal : il n'est possible d'en tirer satisfaction qu'après consommation (en le lisant), mais cette lecture suppose qu'on se le soit approprié (en l'achetant ou par emprunt) préalablement, en aveugle. Or, l'image incitative de cet acte réoriente tout ce qui va suivre, quel qu'il soit, indûment ou pas, sur la cible: ce qu'il fallait que le récit soit pour s'être rendu désirable. Il faut donc que la lecture se fasse en deux temps: une appréhension scopique par le biais d'une image de garde, à laquelle succède une saisie verbale, qui s'y rapporte, comme à sa rive, inlassablement. À cette fascination par les dessous s'oppose, mais s'y conforme l'élaboration angoisseuse, mais éclatante d'une histoire de la révélation. On trouvera, peut-être, seconde objection, que je donne trop de poids à ce dessus de plat auquel les acheteurs de livres disent ne pas prêter plus d'attention qu'il ne faut. Mais il faut se méfier des indifférences et, s'il est sûr que la couverture atteint sans mal depuis toujours des sommets de mauvais goût, il est certain aussi que l'attrait qu'elle formule opère.

Décalage d'une lecture qui se fait donc en deux temps: par avance et provisionnellement à partir de l'image, rétroactivement et pour confirmation, à partir du texte. Il ne reste plus guère autre chose à dire à un livre soigneusement « empaqueté » ou "emboîté » de la sorte que ce que sa couverture illustrée prétend qu'il affirme. Comment sortir un message de l'ornière qui lui a été assignée, comment le faire échapper au rail sur lequel on l'a placé ? Un texte est devenu, en somme, l'otage de la mise en scène des médias mise en place pour le mettre en circuit. Il est donc soumis au signe payant de sa différence marchande. Il existe pour être immédiatement reconduit à la pensée initiatrice, toute imagée qu'elle soit, qui l'ordonne.

Le phénomène que nous décrivons n'implique pas la mise sous tutelle du roman par la télévision, ni que l'image se dispose à envahir tout l'espace du livre ou que nous assistions aux derniers jours de la lecture des ouvrages écrits sur le papier. Il se passe tout simplement que le texte (du roman), saisi par la visualisation couverturielle, se médiatise de son propre mouvement et se projette lui-même, rétroactivement et 
projectivement, sur l'écran que la page de couverture illustrée constitue. Le volume qui le contient est devenu cette petite boîte oblongue sur le couvercle de laquelle nous appliquons, réappliquons et référons, successivement, les images mentales de toute nature que sa lecture déclenche. Ce volume forme une pile d'images qu'attire à soi ou cumule la première d'entre elles; toutes ses pages (les images qu'elles engendrent, les informations qu'elles renferment) viennent se fondre, se combiner, converger sur la première de la série - en haut de la pile -, conservée sur la cornée, dans les tiroirs du souvenir. La couverture imagée de la sorte présenterait quelque chose comme la « scène primitive » de mon désir de lire.

5. Il s'agirait de vérifier maintenant les assertions précédentes sur un cas-type. Je choisis d'examiner, pour ce faire, et bien trop partiellement, j'en suis conscient, mais la tâche est immense, l'ensemble de la production romanesque des Éditions Fleuve Noir s'échelonnant sur presque trente ans, de 1950 à 1978. Toutes les couvertures de cette période - sauf celles qui concerne la science-fiction - sont conçues à partir des maquettes préparées par le dessinateur Michel Gourdon, qui a, comme on sait, imposé son style et sa façon à la maison ${ }^{13}$. Sur un échantillonnage d'une centaine de couvertures, je chercherai à relever les facteurs constitutifs de la puissance fascinatrice de l'image proposée au lecteur. Je considérerai, à cet égard, la couverture comme un super-signe et demanderai ce qu'il convient de donner à voir pour que le désir de regarder se développe et grossisse. Je considère, bien sûr, que les couvertures signées Gourdon ont convenu durant toute cette période et qu'elles ont rempli, alors, sans désemparer, leur office. J'observerai aussi ces couvertures du point de vue de la mise en scène du message auquel elles introduisent. Leur examen m'a conduit à formuler les deux thèses complémentaires suivantes, que je propose dès l'abord, pour faciliter la lecture de ce qui va suivre : la couverture, obturateur du livre, constitue celui-ci en un voir ou, du moins, en un appareil de vision: ce que je lis, finalement, court à sa résolution visuelle; en quoi l'histoire se résorbe, pour mentale qu'elle soit, appelle à sa contemplation et renvoie à la forme initiale prise par celle-ci à la couverture, dont elle est le répondant. Le livre, ensuite, se déchiffre par défaut d'une visualisation qui lui manque; projeté comme insuffisance (il n'est pas conforme à ce qui s'annonce à la couverture), mais repoussant hors lieu la satisfaction du lecteur (il laisse immensément à désirer), il reconduit à l'infini (et sans signification) le geste de lire. Le livre se consomme, tandis qu'en vain nous nous épuisons à cette action.

Voici, en bref, avec quelques exemples, pour chacune des incitations majeures portées par l'image inaugurale, les principaux éléments couverturiels repérés :

31 La couverture pose un voile, un gris, un noir, un fond, une paroi, un obstacle - le signe de l'enfermement et de la difficulté - ce contre quoi le regard va buter, s'enferrer, s'enfoncer, tenir: le mur de briques (Le Paniquard, 1962), la jalousie à lamelles (Impasse noire, 1963), le carrelage noir et blanc (La Part du mal, 1963), la façade d'un building en verre (Trio infernal pour le commander, 1975) servent autant à retenir qu'à lancer la demande. La rétention allume le désir, la barrière qu'il rencontre sollicite son élan, l'objet trouvé dans l'autre espace justifie son action.

La couverture fait considérer un œil, un objectif, un outil de la vision, braqué, circulaire, sur le lecteur, et qui le toise. Je suis regardé regarder ; le regard de l'autre retient mon œil ; sa fixation m'accapare. Une opération scopique figure « dessus » le livre à mon endroit : le héros se trouve au périscope (contact impossible, 1952) : un corps couché, jeté, étendu, mort ou vif, sur une plage, sur la chaussée, darde son regard vers 
celui qui s'approche ; le cerne oculaire de la lune que reprennent et aggravent les deux phares allumés du véhicule qui arrive, saisit le témoin, l'absorbe au cœur de la vision nocturne (Opération pyramides, 1953 - Fig. I -) ; un œil froid, détaché d'un visage mâle, fixé sur l'impétrant, fait contrepoint avec le regard apeuré de la jeune femme en petite tenue, la main sur la poitrine (On demande un minable). La même sollicitation par l'œil se trouve ailleurs, comme bien on pense. Par exemple, à la couverture de l'édition Carré noir d'Au voyeur !, de Carter Brown, en 1979. Cet œil, attentif et primordial, dans la mesure où, cette fois, par exception, il peut saisir son objet, tient dans sa pupille le sein sur lequel le désir initial se fixe et réfléchit sur moi le regard qu'il appelle. Je me tiens aux deux extrémités de la chaîne, en train d'obéir sur le papier au geste irrésistible de mon double.

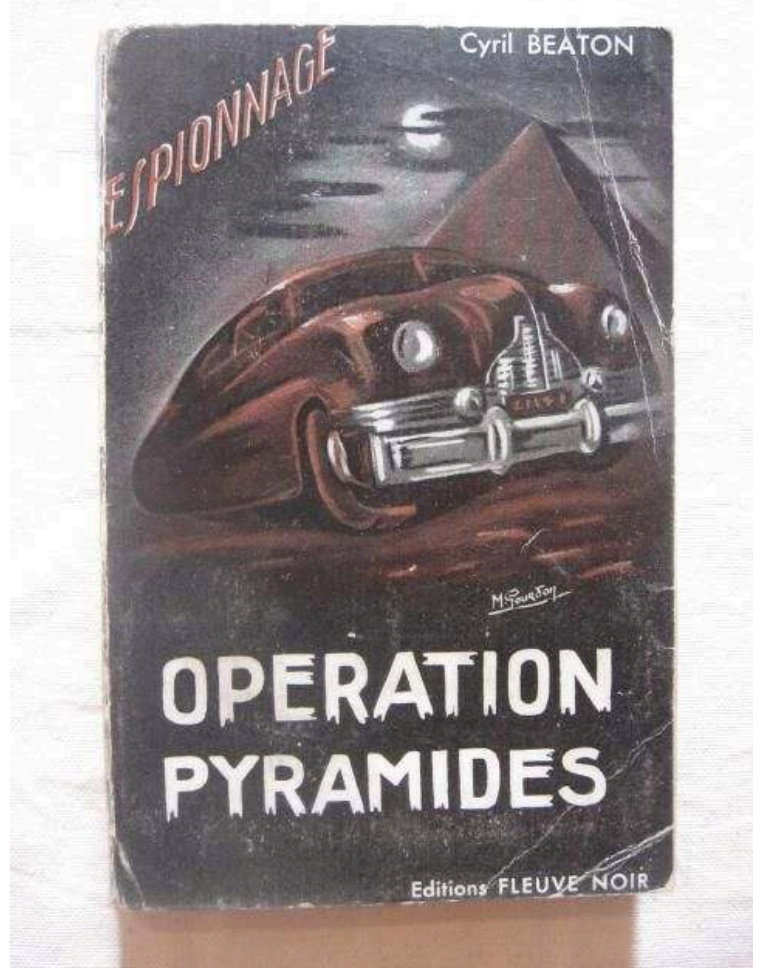

Fig. I : Opération pyramides, Fleuve Noir, 1953

La couverture forme un cadre achevé, un rectangle, une fenêtre, une trappe, un clapet - un objet rond ou carré délimitant et orientant le regard. Elle situe celuici, elle l'adresse, elle lui fait trouver sa cible parmi les objets plus ou moins indifférents que réunit l'histoire. Nous pouvons nous trouver au-devant des carreaux d'une vitre (Périmètre interdit, 1958), dans la chambre et regarder par la baie vitrée dehors (Impasse noire, 1963), dans une pièce sinistre qu'éclaire une fenêtre (Le Transfuge), nous pouvons considérer la suite du drame par le pare-brise de l'automobile où nous avons pris place, derrière le conducteur (Jeux sans loi, 1954 - Fig. 2-). 


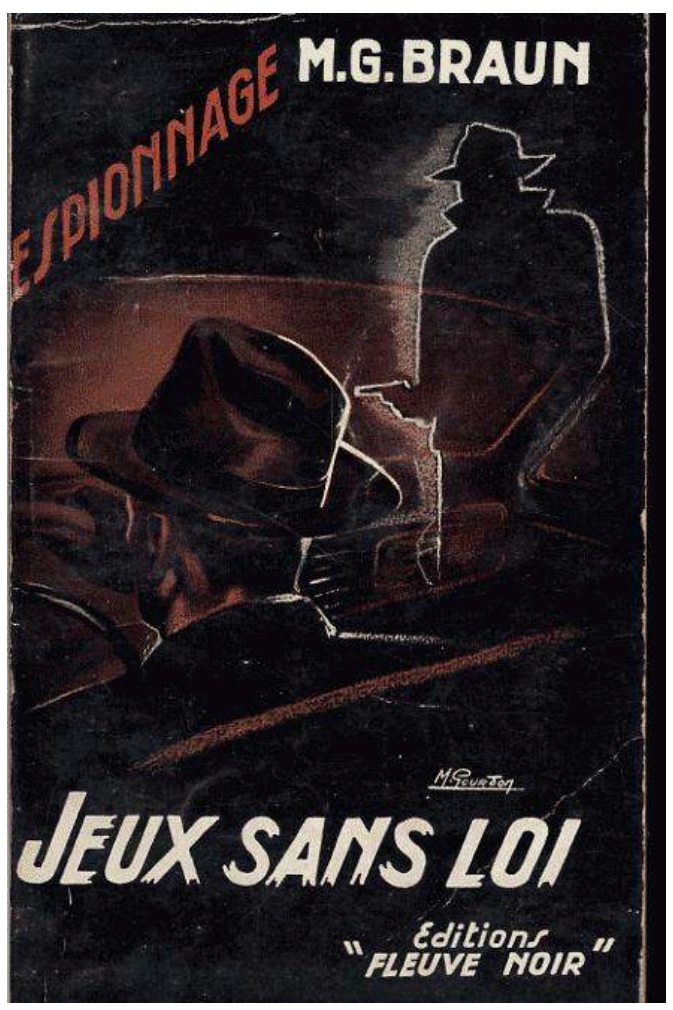

Fig. 2 : Jeux sans loi, Fleuve Noir, 1954

La couverture présente un pli par où s'immiscer, intégrer le récit, correspondre à sa matière. Le dessin qu'elle offre métaphorise la page ouverte, prête à être refermée, ou, inversement, la réticence de l'histoire à résoudre son énigme. Importance de la ligne d'horizon tracée à travers l'image à cet égard. La séparation entre le ciel et la terre, convenablement posée, pose la faille par où la connaissance des choses pourra filtrer - par où gagner le centre inaccessible du récit (Le Double Jeu de Mr. Suzuki, 1965).

La couverture figure la menace, appelle la crainte et donc, très systématiquement, arrête le regard du lecteur sur ce qui l'occasionne. Elle choisit, pour ce faire, par exemple, une arme dressée en train de s'abaisser sur lui. Le trou du canon du pistolet - cet autre œil et ce cerne placide et froid - est visible. Le coup va partir. L'impact est tout près d'être subi (Visages de la nuit, 1955, Impasse noire, 1963). La couverture d'un ouvrage d'une autre collection de poche (de Mickey Spillane), où c'est la belle en habits d'Ève qui brandit l'arme, au bon endroit et de la bonne façon, contre son lecteur, est fameuse. Fameuse aussi l'affiche du comminatoire "Achetez ce livre, sinon j'abats ce chien!». Emblématique encore à cet égard - mais rarissime - la représentation de l'énucléation (La Malédiction des vautours). Ne plus voir constitue ici, en effet, la menace suprême.

La couverture interpelle, parce qu'elle figure un acte de violence. Rendu témoin d'un tel acte, le lecteur tenu là, immobile, cloué au sol, sous la coupe d'une image qui lui est directement assénée, ne peut plus que subir. C'est que la vision fait mal, qu'elle insupporte, que l'objet - à plus forte raison s'il émerge de l'ombre ou tombe subitement du ciel - émotionne ou commotionne celui qui en reçoit la déflagration de plein fouet. L'image couverturielle frappe au corps du lecteur. Le spectacle qu'elle offre, soigneusement dosé, agresse et il agresse pour qu'il subisse. Or, un homme brusqué est un homme gagné. Rien ne résiste à l'entraînement que la peur des images a produit - 
un combat à mains nues (Le Paniquard, 1962), un coup de poing en pleine figure (Périmètre interdit, 1958).

Couverture - couvre et tue ! Un cadavre gît, principalement, au fond du livre. Un objet qui répugne, un objet en trop, une " charogne », quelque chose qu'on ne saurait considérer sans frémir et au contact duquel nous voici confrontés. Comme par un fait exprès, la couverture privilégie ce qu'il convient de ne pas voir - comme le récit frotte le lecteur à l'action qu'il s'agissait de ne pas commettre. La chose en trop, sanglante et répandue, voici ce que le dessin privilégie. Et c'est en toute justice, si j'ose dire, puisque le propre d'une chose en trop, comme d'une obscénité, est de se trouver là, béante, offerte à tous les regards. Et d'insister à partir de ce lieu intermédiaire que constitue la page de seuil du livre encore chez nous, déjà chez elle: l'homme est étendu, un poignard fiché dans le dos (Impasse noire, 1963), la femme repose sans vie, couchée sur le dos (Le Double Jeu de Mr. Suzuki, 1965, Quand le vin est tiré), l'homme est mort (Un Mort sur le sable).

Un processus d'ouverture. Ce que je vois, sur l'image, comme image, figure ouvert impénétrable et cependant "exposé », forcé, violé dans son intimité. Donné, mais repris. Proposé, mais ressaisi. Il y a une promesse à la couverture, une perspective, une permission. L'idée fuse à partir d'une image que le secret sera confié, que le voile tombera, que les pans du manteau seront écartés. Mais aussi que les portes d'ivoire sont toutes prêtes à se refermer. Les figures - qui sont légion - qui représentent ce mouvement sont donc équivoques; tout est bon, d'ailleurs, pour mettre en scène cette pénétrabilité - y compris sa résistance - de la substance romanesque : une clef (La Clef 1965), un couteau, une balafre, une tache de sang sur le corps en train de tomber. Une trace. Une ligne. Un huis. Mais, bien entendu, c'est le corps - féminin - qui offre le plus généreusement de quoi représenter l'accessibilité (et son contraire) à l'imagination scopique. Que ce soit, simplement, par le décolleté (Impasse noire, 1963, Fig. 3 -) ), par l'entrave et les lèvres (Passeport pour la haine, 1964 - Fig. 4 - ), par la bouche sensuellement ouverte (Trio infernal pour le commander; 1975) ou par le jeu infini des vêtements déchirés et des voiles soulevés (Coplan ne lâche pas prise, Jouez serré M.Coplan, Les Mains aux coquilles, L'Ombre d 'Elsa).

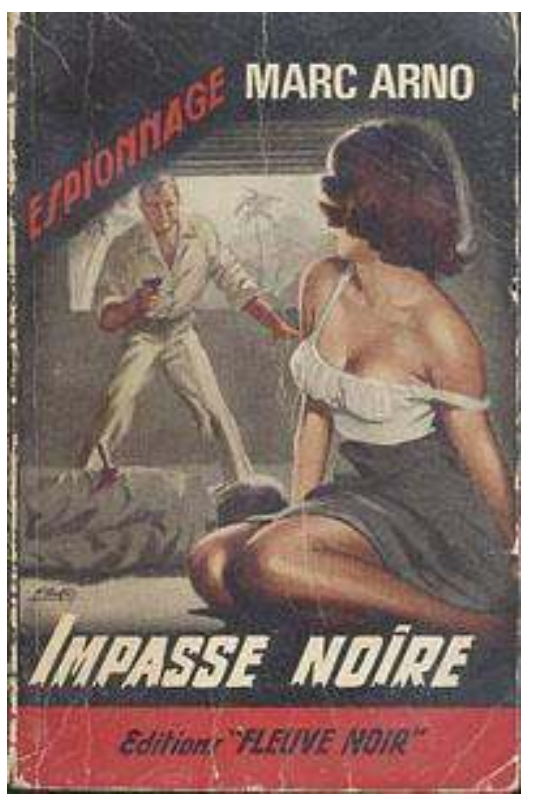

Fig. 3 : Impasse noire, Fleuve noir, 1963 


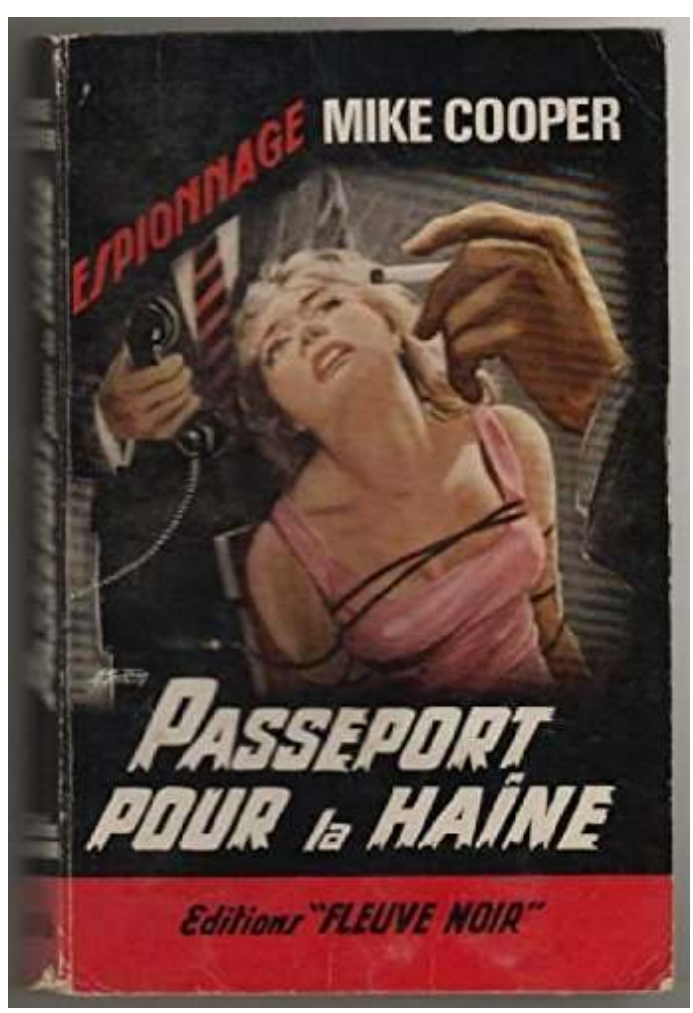

Fig. 4 : Passeport pour la haine, Fleuve Noir, 1964

La couverture rassemble, dans son imagerie, de quoi saisir les corps féminins, de préférence, et le plus déshabillés possible. Il y a comme un impératif catégorique à l'œuvre ici. Comme si le corps manquait. Comme si sa féminité par essence échappait. Comme si en elle se recueillaient tout le désir et tout le sexe. Comme si, bien plus encore, ce n'était que sous la menace d'un coup, d'un couteau, que par le biais d'un enlèvement, comme au temps des Sabines, que contre-gré, grâce à un viol, maintenu par les menottes et la corde, que sa cession pouvait être obtenue. Figure de la poursuite (L'Étrange prédiction - Fig. 5 -) ou de l'étreinte (Le Paniquard, 1962, Schizophrenzie) [sic]), du duel haineux ou de la simple conjonction de deux visages contrastés que tout sépare (Deux Doigts dans la porte, La Nuit sauvage), figure de la capture animale et brutale (Les Chimères où nous regardons s'agiter les bras d'un poulpe gigantesque en direction de la belle endormie) ou figure du tourment et de la torture (La Part du mal, 1964, Passeport pour la haine, 1964, La Main de Dieu), c'est toujours de saisie triomphante et violente qu'il s'agit. Que l'acte sexuel affiche celui de lire et que celui-ci se pense par analogie à la violence que celui-là dégage n'étonnera pas l'observateur: s'il s'agit de déclarer désirable la révélation en quoi le récit est censé consister, il est juste d'user à cette fin des plus imposants appareils - rhétoriques, stéréotypiques et scopiques. 


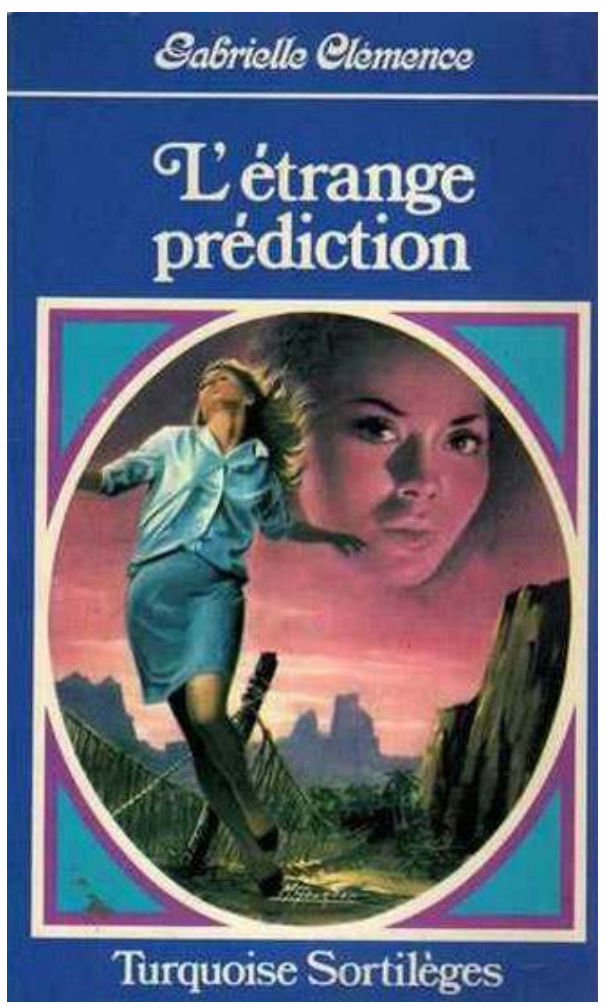

Fig.5 : L'Étrange Prédiction, « Turquoise », Sortilèges, Presses de la Cité, 1981

Étudions d'un peu plus près un cas : la couverture d'un roman policier de James Reach, Martel en tête, paru à la même époque - en 1954 -, aux Presses de la Cité, dans la collection «Un Mystère », Série "Suspense » - Fig. 6 -. Nous constatons, d'abord, que style mis à part, les mêmes causes entraînent les mêmes effets et que les moyens adoptés pour fixer le lecteur, d'une collection à l'autre, ne diffèrent guère. Nous constatons, ensuite, que le motif présenté à la couverture de l'ouvrage - l'étreinte passionnée consentie -, voir la cigarette que la jeune femme tient entre deux doigts de la main gauche et le sourire radieux qu'elle paraît donner à voir - n'a pas de répondant dans l'ouvrage; ce motif est donc purement scopique et non informatif. On peut dire alors que l'image «fausse » infiltre le récit et conforme celui-ci à la nature publicitaire qui est la sienne. On rencontre, par contre, dans l'ouvrage, deux étreintes, différentes de celle dont on vient de parler: la première est celle du meurtrier (un innocent soupçonné de «folie » par sa femme), la seconde est celle du «fou » reconnu coupable. Cette situation complexe aggrave le déficit informatif que l'image de couverture comporte. Cependant, la nature approximative et même sibylline de l'image donnée à voir dès l'abord au lecteur, dans l'iris, ne constitue pas un obstacle à son appréhension de l'intrigue et n'enraie pas le plaisir qu'il en peut tirer. Une image couverturielle vaut pour ce qu'elle ouvre ; sa véracité n'est pas vraiment prise en compte par un lecteur qui reconduira sans mal, approximativement, au coup par coup, ce qu'il lit à ce qu'il a vu préalablement. Le défaut d'exactitude a toute chance de lui échapper - il demandait à la couverture avant de lire le livre autre chose que ce qu'il lui demande après (si même il lui voue encore quelque attention). Par contre, le récit qu'il déchiffre se ressentira de cette prégnance initiale et tout indique que la scène primitive - celle de la violence, celle du drame, celle qui réduit la femme à une proie -, même si nous ne rencontrons pas exactement dans le récit, est engendrée par celui-ci, étant donné l'annonce qui en a été faite liminairement. L'étreinte - la meurtrière étreinte - est le mobile caché du livre 
pensé dans le miroir initial tendu au lecteur par l'image et ce qui a été vu prime, décidément, ce qui pourra en être raconté.

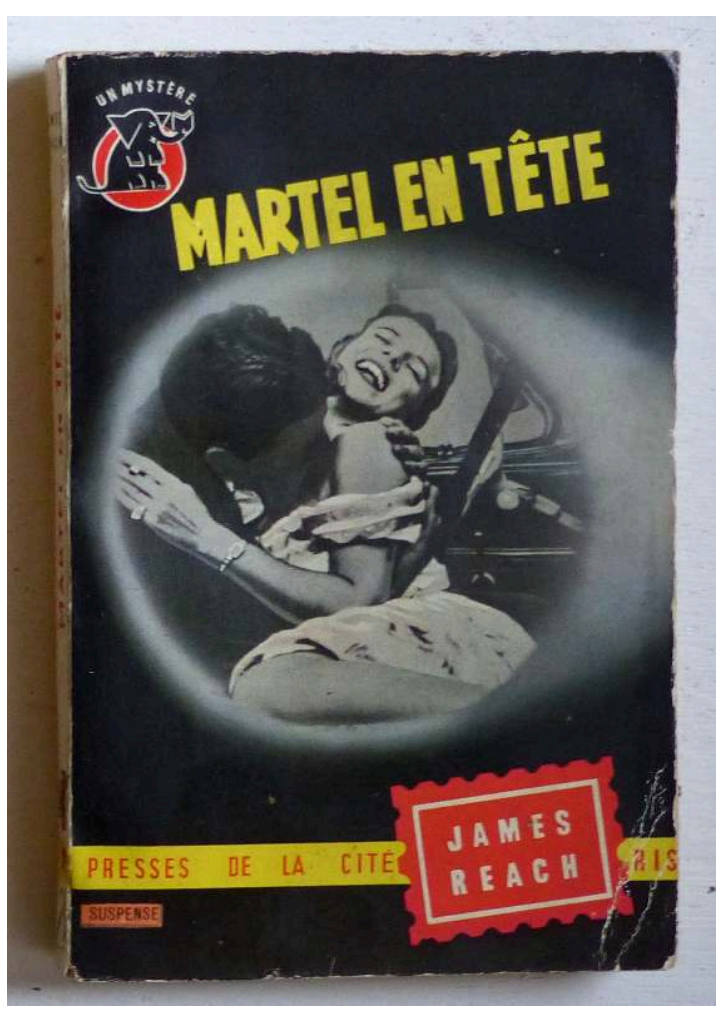

Fig. 6 : Martel en tête, James Reach, « Suspense », Presses de la Cité ; 1954

Que cette vue prophétique soit ici photographique ne laissera pas indifférent: la photographie a été longtemps, est encore peut-être, cette façon de dire que ce qu'on regarde existe en effet.

Il n'empêche : la couverture illustrée ne vise aucun réel, l'image qu'elle offre ne possède aucune vraisemblance, n'articule aucune vérité, ne fixe aucun événement, ne correspond à aucune scène possible ou sensible. On ne l'entend bien cet "au-dedans ", mentalement, déréalisée et sans référent. C'est ce qui lui permet de remplir son office : plus elle s'affranchit des données du récit qui va suivre, plus elle pourra aiguiller le regard sur ce que celui-ci ne pouvait pas dire. On peut désirer qu'une couverture soit muette, vierge de tous bruits, de tout sens et de tous signes - blanche. Même ainsi dépouillée, son vertige sera grand ${ }^{14}$.

\section{NOTES}

1. Prestigieux avec le recul!

2. Même les « grands noms » subissent cette éclipse.

3. Le Roman du quotidien. Lecteurs et lectures populaires à la Belle Époque, Seuil-Points, 2000, p. 127-128. 
4. Seuils, Seuil, 1987, p. 26-27.

5. Ibid., p. 376.

6. Corti, 2001, p. 245 et suivantes.

7. L'Image comme couverture et ouverture. Dans Mireille Ribière, Jan Baetens (éd.), Time, Narrative and the fixed Image. Temps, Narration et Image fixe, Amsterdam, Rodopi, 2001, p. 81-90.

8. Il a en vue les couvertures des collections policières modernes.

9. Cette étude constitue le chapitre 4 de son livre Fictions, Énigmes, Images, publié en 2001 aux Pulim, p. 59-73.

10. Je n'insiste pas sur les parades couverturielles auxquelles Le Rocambole, bulletin de l'Association des Amis du Roman populaire, et les ouvrages publiés à l'enseigne des éditions Encrage nous ont accoutumés: la couverture fascine, cette fascination, pour un peu, ferait oublier le texte auquel elle introduit, Tout se passe comme si, à distance du moins, elle pouvait se suffire à elle-même en toute autonomie. C'est là un phénomène dont on ne peut pas ne pas tenir compte.

11. Cela, en prenant les choses par le petit bout, pragmatique, de la lorgnette. Voir Claude Maggiori, "Création d'images. Neuf histoires de pub », dans Autrement, nº 84 (novembre 1986),

p. 24.

12. Bois dormant et autres poèmes en prose, Gallimard/Poésie. 2002. p. 143.

13. Sur l'impressionnante activité de cet artiste qui pense, d'ailleurs, livrer un travail de peintre - treize couvertures par mois, plus de cinq mille dessins au total -, voir G.J. Arnaud, Trente et un ans de roman populaire, Office municipal de la culture et des arts, La Seyne-sur-Mer, 1983 (R. Bonaccorsi éditeur). Gourdon travaille sur des indications extrêmement peu s: la description approximative des personnages, deux ou trois passages supposés caractéristiques du livre. L'indigence même de la «documentation » dont il se sert facilite le va et vient entre l'image de couverture qui est censée en émaner et le texte d'un roman que l'illustrateur n'a pas à lire.

14. On rapportera les analyses proposées ici à La Couverture d'images. Dans J.E. Müller (éd.), Texte et médialité, MANA 7 (Publications du département de langues et littératures romanes de l'université de Mannheim, 1987, p. 127-189), dont le cadre est plus large et la perspective plus nettement historique.

\section{AUTHOR}

\section{CHARLES GRIVEL}

Université de Mannheim 